\title{
A lower bound on the probability that a binomial random variable is exceeding its mean
}

\author{
Christos Pelekis* Jan Ramon ${ }^{\dagger}$
}

April 22, 2016

\begin{abstract}
We provide a lower bound on the probability that a binomial random variable is exceeding its mean. Our proof employs estimates on the mean absolute deviation and the tail conditional expectation of binomial random variables.
\end{abstract}

Keywords: lower bounds; binomial tails; tail conditional expectation; mean absolute deviation; hazard rate order

\section{Prologue, related work and main result}

Given a positive integer $n$ and a real number $p \in(0,1)$, we denote by $\operatorname{Bin}(n, p)$ a binomial random variable of parameters $n$ and $p$. Here and later, given two random variables $X, Y$, the notation $X \sim Y$ will indicate that $X$ and $Y$ have the same distribution. The main purpose of this note is to illustrate that estimates on the mean absolute deviation of a binomial random variable yield a lower bound on $\mathbb{P}[X \geq n p]$, where $X \sim \operatorname{Bin}(n, p)$. It should come as no surprise that there exists general machinery that can be employed to such a problem. For example, using Cauchy-Schwartz inequality one can show that, for any random variable $Z$ whose mean equals zero, it holds

$$
\mathbb{P}[Z \geq 0] \geq \frac{1}{4} \cdot \frac{\{\mathbb{E}[|Z|]\}^{2}}{\mathbb{E}\left[Z^{2}\right]}
$$

and the bound can be improved further under information on higher moments (see Veraar [11]). If we now let $Z=X-n p$, where $X \sim \operatorname{Bin}(n, p)$, then (1) provides a lower bound

\footnotetext{
*Informatics Section, KU Leuven, Celestijnenlaan 200A, 3001, Belgium; Email: pelekis.chr@gmail.com

†'Informatics Section, KU Leuven, Celestijnenlaan 200A, 3001, Belgium; Email: Jan.Ramon@cs.kuleuven.be
} 
on the probability that a binomial random variable is exceeding its expectation that is expressed in terms of the second moment of $X$ and (the square of) its mean absolute deviation, i.e., $\mathbb{E}[|X-n p|]$. Notice that the bound given by (1) is less than $\frac{1}{4}$, for any zero-mean random variable $Z$. This bound, being rather general, does not use any of the properties of the binomial law. Moreover, it is well known (see Kaas and Burhman [6]) that a median of a binomial random variable of parameters $n$ and $p$ is larger than or equal to $\lfloor n p\rfloor$, the largest integer that is less than or equal to $n p$. Hence $\mathbb{P}[\operatorname{Bin}(n, p) \geq\lfloor n p\rfloor] \geq \frac{1}{2}$ which suggests that, in the case of binomial distributions, there may be space for improvement upon the bound provided by (1). In a recent article Greenberg and Mohri [4] provide the estimate

$$
\mathbb{P}[\operatorname{Bin}(n, p) \geq n p]>\frac{1}{4}, \text { for } p \geq \frac{1}{n} .
$$

It is remarkable that this bound, despite the fact that it is rather intuitive and has been used several times in the literature (see [4]), appears to have been formally verified quite recently. Notice that the inequality is strict. A weaker version of this bound has been reported by Rigollet and Tong [8] and reads as follows:

$$
\mathbb{P}[\operatorname{Bin}(n, p) \geq n p] \geq \min \left\{p, \frac{1}{4}\right\}, \text { for } p \leq \frac{1}{2} .
$$

Such bounds are of particular interest in machine learning and related areas where they are used in the analysis of the, so-called, relative deviation bounds and generalisation bounds. Briefly, both bounds (2) and (3) are obtained using the observation

$$
\mathbb{P}[\operatorname{Bin}(n, p) \geq n p] \geq \mathbb{P}[\operatorname{Bin}(n, k / n) \geq k+1],
$$

where $k$ is the unique positive integer such that $k<n p \leq k+1$, and the problem is reduced to the one of estimating from below the tail of a binomial random variable whose mean is an integer. In this note we apply a similar idea to the tail conditional expectation of a binomial random variable. This allows to obtain a refined version of the aforementioned bounds, when the variance is larger than 8 . More precisely, we have the following.

Theorem 1.1. Fix a positive integer $n \geq 2$ and a real number $p \in\left[\frac{1}{n}, 1-\frac{1}{n}\right]$. Let $X \sim \operatorname{Bin}(n, p)$. Then

$$
\mathbb{P}[X \geq n p] \geq \frac{1}{2 \sqrt{2}} \cdot \frac{\sqrt{n p(1-p)}}{1+\sqrt{n p(1-p)+1}} .
$$

Since the function $f(x)=\frac{\sqrt{x}}{1+\sqrt{x+1}}$ is increasing it is not difficult to see, by investigating the inequality $\sqrt{2 x} \geq 1+\sqrt{x+1}$, that the previous bound is larger than $\frac{1}{4}$, when the parameters $n, p$ satisfy $n p(1-p) \geq 8$. We prove Theorem 1.1 in the next section. Our article ends with Section 3 in which we sketch a proof of a lower bound on the probability that a Poisson random variable is exceeding its mean. 


\section{Proof of Theorem 1.1}

In this section we prove our main result. We begin by collecting certain results on the median, the mean absolute deviation and the tail conditional expectation of a binomial random variable.

Lemma 2.1. Let $X \sim \operatorname{Bin}(n, p)$ and suppose that $n p$ is an integer. Then

$$
\mathbb{P}[X \geq n p]>\frac{1}{2}
$$

Proof. This is a well known result that can be found in several places. See, for example, Jogdeo et al. [5], Kaas et al. [6], or Siegel [10, Theorem 2.2].

This result yields an upper bound on the tail conditional expectation of a binomial random variable whose mean is an integer.

Lemma 2.2. Let $X \sim \operatorname{Bin}(n, p)$ and suppose that $n p$ is an integer. Then

$$
\mathbb{E}[X \mid X \geq n p]<n p+\sqrt{n p(1-p)} .
$$

Proof. Clearly, we have

$$
\mathbb{E}[\max \{0, X-n p\}]=\mathbb{P}[X \geq n p] \cdot \mathbb{E}[X-n p \mid X \geq n p]
$$

which, in view of Lemma2.1, implies

$$
\mathbb{E}[X-n p \mid X \geq n p]<2 \cdot \mathbb{E}[\max \{0, X-n p\}] .
$$

To simplify notation, set $Z^{+}=\mathbb{E}[\max \{0, X-n p\}]$ and $Z^{-}=\mathbb{E}[\min \{0, X-n p\}]$. Then $Z^{+}-Z^{-}=\mathbb{E}[X-n p]=0$ as well as $Z^{+}+Z^{-}=\mathbb{E}[|X-n p|]$ and therefore, upon adding the last two equations, we conclude

$$
\mathbb{E}[\max \{0, X-n p\}]=\frac{1}{2} \cdot \mathbb{E}[|X-n p|] .
$$

Hence we have $\mathbb{E}[X-n p \mid X \geq n p]<\mathbb{E}[|X-n p|]$. The estimate

$$
\mathbb{E}[|X-n p|] \leq \sqrt{\mathbb{E}\left[|X-n p|^{2}\right]}
$$

finishes the proof.

Notice that the previous result employs an upper bound on the mean absolute deviation. The proof of Theorem 1.1 will require a corresponding lower bound. 
Lemma 2.3. Fix positive integer $n \geq 2$ and let $X \sim \operatorname{Bin}(n, p)$. If $p \in\left[\frac{1}{n}, 1-\frac{1}{n}\right]$ then

$$
\mathbb{E}[|X-n p|] \geq \sqrt{\frac{n p(1-p)}{2}} .
$$

Proof. See Berend and Kontorovich [1].

Recall (see [9]) that an integer-valued random variable $X$ is said to be smaller than the integer-valued random variable $Y$ in the hazard rate order, denoted $X \leq_{h r} Y$, if

$$
\frac{\mathbb{P}[X \geq k]}{\mathbb{P}[X \geq k+1]} \geq \frac{\mathbb{P}[Y \geq k]}{\mathbb{P}[Y \geq k+1]}, \text { for all } k=1,2, \ldots
$$

Recall also that $X$ is said to be smaller than $Y$ in the likelihood ratio order, denoted $X \leq_{l r} Y$, if $\frac{\mathbb{P}[X=k]}{\mathbb{P}[Y=k]}$ is decreasing in $k$. It is known (see [9. Theorem 1.C.1]) that if $X \leq_{l r} Y$ then $X \leq_{h r} Y$.

Lemma 2.4. Fix a positive integer $n$ and let $p, q \in(0,1)$ be such that $p<q$. Suppose that $X_{p} \sim \operatorname{Bin}(n, p), X_{q} \sim \operatorname{Bin}(n, q)$, and fix a positive integer $k \in\{0,1, \ldots, n\}$. Then

$$
\mathbb{E}\left[X_{p} \mid X_{p} \geq k\right] \leq \mathbb{E}\left[X_{q} \mid X_{q} \geq k\right] .
$$

Proof. This is a well known result (see [2, 7]). We include some details of the proof for the sake of completeness. Notice that the result will follow once we show that

$$
\mathbb{P}\left[X_{p} \geq k+t \mid X_{p} \geq k\right] \leq \mathbb{P}\left[X_{q} \geq k+t \mid X_{q} \geq k\right], \text { for all } t \in\{1, \ldots, n-k\} .
$$

Fix $t \in\{1, \ldots, n-k\}$ and note that it is enough to show that the function $f(p)=\frac{\mathbb{P}\left[X_{p} \geq k+t\right]}{\mathbb{P}\left[X_{p} \geq k\right]}$, where $p \in(0,1)$, is increasing in $p$. Now notice that

$$
\frac{\mathbb{P}\left[X_{p} \geq k+t\right]}{\mathbb{P}\left[X_{p} \geq k\right]}=\prod_{j=0}^{t-1} \frac{\mathbb{P}\left[X_{p} \geq k+j+1\right]}{\mathbb{P}\left[X_{p} \geq k+j\right]}
$$

which, in turn, implies that it is enough to show that, for $j=0, \ldots, t-1$, it holds

$$
\frac{\mathbb{P}\left[X_{p} \geq k+j+1\right]}{\mathbb{P}\left[X_{p} \geq k+j\right]} \leq \frac{\mathbb{P}\left[X_{q} \geq k+j+1\right]}{\mathbb{P}\left[X_{q} \geq k+j\right]}, \text { for } p<q .
$$

In other words, it is enough to show that $X_{p} \leq_{h r} X_{q}$. The later can be concluded either by induction on $n$ (see [2, Proposition 1.1]) or from the fact (see [7]) that $X_{p} \leq_{l r} X_{q}$.

We now have all the necessary tools to prove our main result. If $x$ is a positive real, we denote by $\lceil x\rceil$ the minimum integer that is larger than or equal to $x$ and we set $[[x]]=\lceil x\rceil-x$. 
Proof of Theorem 1.1. In case $n p$ is an integer, Lemma 2.1 implies that $\mathbb{P}[X \geq n p]>1 / 2$ and therefore the result holds true. So we may assume that $n p$ is not an integer. Let $k$ be the unique positive integer such that $k<n p<k+1$. Since $p \in\left[\frac{1}{n}, 1-\frac{1}{n}\right]$ Lemma 2.3 implies that

$$
\mathbb{P}[X \geq n p]=\frac{1}{2} \cdot \frac{\mathbb{E}[|X-n p|]}{\mathbb{E}[X-n p \mid X \geq n p]} \geq \frac{1}{2 \sqrt{2}} \cdot \frac{\sqrt{n p(1-p)}}{\mathbb{E}[X-n p \mid X \geq n p]}
$$

and so it is enough to find an upper bound on $\mathbb{E}[X-n p \mid X \geq n p]$. Now notice that the assumption that $n p$ is not an integer implies

$$
\mathbb{P}[X \geq n p]=\mathbb{P}[X \geq k+1] \quad \text { as well as } \quad \mathbb{E}[X \mid X \geq n p]=\mathbb{E}[X \mid X \geq k+1] .
$$

Let $Y \sim \operatorname{Bin}\left(n, \frac{k+1}{n}\right)$ and note that $Y$ is a binomial random variable whose mean is an integer. Since $p<\frac{k+1}{n}$, Lemma 2.4 yields

$$
\mathbb{E}[X \mid X \geq k+1] \leq \mathbb{E}[Y \mid Y \geq k+1] .
$$

Since $\mathbb{E}[Y]$ is an integer, Lemma 2.2 yields

$$
\begin{aligned}
\mathbb{E}[Y \mid Y \geq k+1] & \leq k+1+\sqrt{(k+1)\left(1-\frac{k+1}{n}\right)} \\
& =n p+[[n p]]+\sqrt{(n p+[[n p]])\left(1-p-\frac{[[n p]]}{n}\right)} \\
& =n p+[[n p]]+\sqrt{n p(1-p)+[[n p]](1-2 p)-\frac{[[n p]]^{2}}{n}} \\
& \leq n p+1+\sqrt{n p(1-p)+1-2 p}
\end{aligned}
$$

and so

$$
\mathbb{E}[X-n p \mid X \geq k+1] \leq 1+\sqrt{n p(1-p)+1-2 p} .
$$

Putting all the above together, we see that

$$
\mathbb{P}[X \geq n p] \geq \frac{1}{2 \sqrt{2}} \cdot \frac{\sqrt{n p(1-p)}}{1+\sqrt{n p(1-p)+1-2 p}}
$$

and the result follows.

\section{Concluding remarks}

So far we obtained a lower bound on the probability that a binomial random variable is exceeding its mean. Our proof combines the identity

$$
\frac{1}{2} \cdot \mathbb{E}[|X-\mu|]=\mathbb{P}[X \geq \mu] \cdot \mathbb{E}[X-\mu \mid X \geq \mu], \text { where } \mu=\mathbb{E}[X]
$$


with a lower bound on the mean absolute deviation (MAD) and an upper bound on the tail conditional expectation (TCE). Notice that (4) holds true for any random variable and therefore it may be employed whenever one can estimate the MAD from below and the TCE from above. For example, it is known (see [3, Example 1]) that $\mathbb{E}\left[\left|P_{\lambda}-\lambda\right|\right]=$

$2 \lambda \frac{e^{-\lambda} \lambda^{\lfloor\lambda\rfloor}}{[\lambda ! !}$, where $P_{\lambda}$ is a Poisson random variable of mean $\lambda$. It is also known that when $\lambda$ is an integer then a median of a Poisson random variable is equal to its mean (see [6, Section 2]). Therefore, when $\lambda$ is an integer, a similar argument as the one used in the proof of Theorem 2.2 yields

$$
\mathbb{E}\left[P_{\lambda} \mid P_{\lambda} \geq \lambda\right] \leq \lambda+\sqrt{\lambda}
$$

When $\lambda$ is not an integer, standard results on the likelihood ratio order of Poisson random variables (see [9. Section 1.C] and [7]) imply that

$$
\mathbb{E}\left[P_{\lambda} \mid P_{\lambda} \geq \lambda\right]=\mathbb{E}\left[P_{\lambda} \mid P_{\lambda} \geq k\right] \leq \mathbb{E}\left[P_{k} \mid P_{k} \geq k\right],
$$

where $k$ is the smallest integer that is larger than $\lambda$ and $P_{k} \sim \operatorname{Poi}(k)$. Therefore a similar argument as the one used in the proof of Theorem 1.1, combined with the Stirling estimate $\lfloor\lambda\rfloor ! \leq e \lambda^{\lfloor\lambda\rfloor+\frac{1}{2}} e^{-\lfloor\lambda\rfloor}$, yields the bound

$$
\mathbb{P}\left[P_{\lambda} \geq \lambda\right] \geq \frac{2}{e^{\lambda-\lfloor\lambda\rfloor+1}} \cdot \frac{\sqrt{\lambda}}{1+\sqrt{\lambda+1}}, \text { for } P_{\lambda} \sim \operatorname{Poi}(\lambda) .
$$

Finally, let us remark that most lemmata from Section2 2 appear to be extendable to sums of independent and heterogeneous Bernoulli random variables. However, we were not able to provide an analogue of Lemma 2.3 for this case. That is, we were unable to find a sharp lower estimate on $\mathbb{E}\left[\left|\sum_{i} B_{i}-\sum_{i} p_{i}\right|\right]$, where each $B_{i}$ is a $0 / 1$ Bernoulli random variable of mean $p_{i}$. Such a lower estimate could in turn provide a lower bound on $\mathbb{P}\left[\sum_{i} B_{i} \geq \sum_{i} p_{i}\right]$ and we hope that we will be able to report on that matter in the future.

\section{References}

[1] D. Berend, A. Kontorovich. A sharp estimate of the binomial mean absolute deviation, Statistics \& Probability Letters 83, 1254-1259, (2013).

[2] E. Broman, T. van de Brug, W. Kager, R. Meester. Stochastic domination and weak convergence of conditioned Bernoulli random variables, ALEA Latin Amer. J. Probab. Math. Stat. 9, no. 2, 403-434, (2012).

[3] P. Diaconis, S. Zabell. Closed form summation for classical distributions: variations on a theme of de Moivre, Statistical Science 6 (3), 284-302, (1991). 
[4] S. Greenberg, M. Mohri. Tight lower bound on the probability of a binomial exceeding its expectation, Statistics \& Probability Letters 86, 91-98, (2014).

[5] K. Jogdeo, S. Samuels. Monotone convergence of binomial probabilities and a generalisation of Ramanujan's equation, The Annals of Mathematical Statistics 39, 1191-1195, (1968).

[6] R. Kaas, J.M. Burhman. Mean, median, mode in the binomial distribution, Statistica Neerlandica 34 (1), 13-18, (1980).

[7] A. Klenke, L. Mattner. Stochastic ordering of classical discrete distributions, Advances in Applied Probability 42, no. 2, 392-410, (2010).

[8] P. Rigollet, X. Tong. Neyman-Pearson classification, convexity and stochastic constraints, Journal of Machine Learning Research 12, 2831-2855, (2011).

[9] M. Shaked, G.J. Shanthikumar. Stochastic Orders, Springer, New York, 2007.

[10] A. Siegel. Median bounds and their applications, Journal of Algorithms 38, Issue 1, 184-236, (2001).

[11] M. Veraar. A note on optimal probability lower bounds for centered random variables, Colloquium Mathematicum 113, no. 2, 231-240, (2008). 